\title{
Seroprevalence of Hepatitis B, Hepatitis C, and human immuno- deficiency virus and their risk factors in adolescents in East Sik- kim, a Himalayan state of India
}

\author{
Luna Adhikari' ${ }^{1}$ Ashish Pradhan², Dheeraj Khatri ${ }^{3}$
}

\section{Luna Adhikari ${ }^{1}$, Ashish \\ Pradhan $^{2}$, Dheeraj Khatri ${ }^{3}$}

\section{'Department of Microbiology, Sikkim Manipal Institute of Medical Sciences, Tadong, Gangtok, Sikkim, India. 2Department of Pediatrics, Sikkim Manipal Institute of Medical Sciences, Tadong, Gangtok, Sikkim, India. ${ }^{3}$ Deptartment of Medicine, Sikkim Manipal Institute of Medical Sciences, Tadong, Gangtok, Sikkim, India.}

\section{Correspondence}

Dr. Luna Adhikari, MD (Microbiology), Professor,

'Department of Microbiology, Sikkim Manipal Institute of Medical Sciences, Tadong, Gangtok, Sikkim, India.

Email: lunashim@hotmail.com

Mobile Number: 0091-9434864772

\section{History \\ - Submission Date: 03-09-15; \\ - Review completed: 18-06-16; \\ - Accepted Date: 22-06-16.}

DOI : 10.5530/ijmedph.2016.3.10

Article Available online

http://www.ijmedph.org/v6/i3

\section{Copyright}

(c) 2016 Phcog.Net. This is an open-access article distributed under the terms of the Creative Commons Attribution 4.0 International license.

\begin{abstract}
Background: Sikkim is a northeastern state of India, located in the Himalayas with migratory population from neighboring states and Nepal. Although there is high prevalence of human immunodeficiency virus (HIV) and Hepatitis $B$ and $\mathrm{C}$ virus (HBV and $\mathrm{HCV}$ ) in other northeastern states of India, currently, this information is lacking in Sikkim's adolescent population, which inspired the researchers to conduct this study.

Methods: This is a cross-sectional study with 490 adolescent participants between the age group of13-20 years. Schools/colleges and the participants were randomly selected. Blood samples of selected participants were collected after their interview and were tested for antiHIV, hepatitis B surface antigen, and anti-HCV.

Findings: Mean age of the participants was 16.96 years. Only $1(0.2 \%)$ participant was positive for HBsAg, and none was positive for anti-HIV and anti-HCV. About 92 (18.78\%), 90 (18.37\%), 79 (16.12\%), and 12 (2.45\%) participants had respectively undergone tattoo/body piercing, invasive (medical) procedure, shared personal items, and had received blood transfusion but none of them provided information on their use of injectable drugs. A total of $33(6.73 \%)$ participants expressed their involvement in heterosexual relation, of which 29 $(29 / 215,13.49 \%)$ were boys and $4(4 / 275,1.45 \%)$ were girls $(p<0.01)$. Among them, 13/13/33, $39.39 \%)$ participants expressed their involvement with multiple sexual partners and 16 (16/33, $48.49 \%$ ) participants had taken protective measures. None of the girls provided history about their partner taking any protective measures.

Interpretation: To the best of our knowledge, this is the first study reporting on the prevalence of HIV, HBV, and HCV in the adolescent population of Sikkim. Our results indicate that there is a very low prevalence of HBV and absence of HIV and HCV infections in the studied population. However, the studied population is at risk of acquiring these infections and therefore necessary steps must be taken to prevent the transmission of these viruses.

Funding: Indian Council of Medical Research.
\end{abstract}

Key words: HIV, HBV, HCV.

\section{INTRODUCTION}

The United Nations Program on human immunodeficiency virus (HIV)/acquired immunodeficiency syndrome (AIDS) (UNAIDS) and the World Health Organization (WHO) jointly declared AIDS as one of the most destructive pandemics in the recorded history. ${ }^{1}$ Asia is the secondworst affected region after sub-Saharan Africa, with 4.9 million people living with $\operatorname{HIV}\left(15 \%\right.$ of the global total). ${ }^{2}$ The economic consequences of AIDS will force an additional 6million households in Asia into poverty by 2015 unless national responses are significantly strengthened. ${ }^{3}$ The three northeastern states of India namely, Manipur, Mizoram, and Nagaland have only 3\% of the country's total population. Unfortunately, these states harbor $25 \%$ of India's HIV-positive cases. ${ }^{4}$ In 1989, no HIV infections had been detected in Manipur, but in February 1990, five HIV-positive cases were reported, and by May 1991, 1,263 HIV-positive cases had been reported. Considering that Manipur makes up $<1 \%$ of the country's total population and only 5,131 HIV-positive cases had been reported in all of India during the same period, this came as a shocking news. ${ }^{5}$

Infection due to hepatitis B virus (HBV) is a global public health concern. Nearly 2 billion people in the world have been infected by HBV. Among them, nearly 350 million people have been chronically infected. ${ }^{6,7}$ In India, a country that is yet to adopt an organized HBV-prevention program, nearly 3-4\% of the population is infected by HBV. ${ }^{8}$

Infection due to hepatitis $\mathrm{C}$ virus (HCV) is another leading cause of chronic liver diseases. About 2-4 million people may be chronically infected with $\mathrm{HCV}$ in the United States, about 5-10 million in Europe, and about 12 million in India, and sadly most of them are unaware about their infection. ${ }^{9}$

A large population and the absence of effective surveillance and prevention programs could increase the burden of these infections and its consequences in India in the years to come. In this perspective, in India, a blood-borne viral disease epidemiologi- 
cal study becomes relevant in both national and international context because of the possibility that India may soon harbor the largest bloodborne viral infection pool in the world.

Sikkim, one of the eight northeast states of India, is located in the Himalayas surrounded by China, Bhutan, Nepal, and West Bengal (a state of India). Sikkim has a population of $607,688 .{ }^{10}$ Recent developmental activities in Sikkim has led to a huge population of migratory people, chiefly laborers from Nepal and also from West Bengal and Bihar states of India. To the best of our knowledge, there is no documented data on the prevalence of these infections in adolescents of Sikkim. Lack of information on such life-threatening infections in highly vulnerable adolescent population of Sikkim inspired the researchers to conduct this study to determine the seroprevalence of these infections in the adolescent population of east Sikkim.

\section{METHODS}

\section{Study design and participants}

This is a cross-sectional study conducted from June 2012 to June 2013. This study was approved by the Departments of Human Resource Development and Health Care, Human Service, and Family Welfare, Government of Sikkim, India and by Research Protocol Evaluation Committee and Institutional Ethics Committee, Sikkim Manipal Institute of Medical Sciences, India. We recruited participants from seven schools and seven colleges across east Sikkim. Students from classes 7 to 12 and first year of college were included in this study. The eligibility criteria were students between the age group of 13-20 years and indigenous inhabitants of Sikkim. We excluded students under 18 years whose parent/parents did not give consent and those who refused to participate in the study. We also excluded participants above 18 years who did not give consent. The purpose and objective of this study was explained to the selected students in both English and in local language. Students were informed that they would participate in the study voluntarily, and that no researchers would force any of the students to take part in the study, and that they have the right to withdraw from the study any time they desire even after giving consent without penalty. They were also informed about their confidentiality and that their data generated would be used only for research work. Informed consent was obtained from all the participants. For participants with age $<18$ years, parental consent was obtained.

Sample size: Sample size was estimated using the formula $n=Z^{2}(1-d) / d^{2}$, where $n=$ sample size, $Z=Z$ statistic for a level of confidence, $P=$ expected prevalence or proportion (in proportion of one), $d=$ precision ( $d$ was taken as half of $P$ because reference $P$ was below 0.1 (10\%). Prevalence of $\mathrm{HIV}$ in adolescents in India (8.6\%) as reported by All India Institute of Medical Sciences, Delhi was taken as the reference value. ${ }^{11}$ Based on this data, the sample size obtained was 163 . Because the prevalence of other two blood-borne viruses in adolescent population in India is unknown, three times of the calculated sample size was taken, that is, 489. To uniformly distribute the sample size across all the seven schools and seven colleges, the final sample size was estimated to be 490 .

\section{Randomization}

Seven schools and seven colleges were selected randomly from the official list of schools and colleges of the state. For selection of the participants, we randomized the class according to the class attendance register (classes 7-12 and first year of college). From each class, first 10students were considered for participation in the study. The school/college that had less than 10participants in any of the aforementioned classes was excluded from the study, and the school/college that was immediately below the excluded school/college in the randomization list was included in the study. If the student who was included in the randomization list refused to take part in the study, then that student was excluded, and the student below the excluded student in the randomization list was included. A code number was assigned to each student who took part in the study for their identification, which was kept confidential.

\section{Procedures}

After an informal introduction with each participant, a set of pretested questions were asked to explore information regarding mode of transmission of blood-borne infections. From each participant, $5 \mathrm{ml}$ of blood sample was collected by using sterile disposable needle and syringe by taking universal precautions. The serum was separated from the blood samples. Anti-HIV in the serum sample was tested as per National AIDS Control Organization (NACO) guidelines (SD (Bioline) HIV1/2 3.0; Sensitivity $100 \%$ and Specificity $100 \%) .{ }^{12}$ Presence of hepatitis B virus surface antigen (HBsAg) in the serum sample was tested by a rapid test based on the principal of immune chromatography ( $\mathrm{SD}$ (Bioline)HBsAg; Sensitivity $100 \%$ and Specificity $100 \%$ ). The presence of anti-HCV in the serum sample was detected by a fourth generation ELISA (Monalisa ${ }^{\mathrm{TM}}$ HCV Ag-Ab ULTRA; Sensitivity 100\% and Specificity 100\%). The data generated was maintained in a register according to code number, age, sex, and results of the test.

\section{Statistical analysis}

The data were analyzed by chi-square test. The chi-square value was interpreted at 10 degrees of freedom. Statistical significance was assessed at $p<0.05$ (95\% confidence interval).

\section{RESULTS}

A total of 490 participants were included in the study. Table 1 shows the distribution of age and sex of the participants. Their mean age was 16.96 years. Only $1(0.2 \%)$ participant was positive for HBsAg. None of the participant was positive for anti-HIV and anti-HCV.

About 92 (18.78\%), 90 (18.37\%), 79 (16.12\%), and 12 (2.45\%) participants provided history of undergoing tattoo/body piercing, invasive (medical) procedure, sharing of personal items, and of receiving blood transfusion, respectively. None of the participants provided history of using injectable drugs, but $4(0.82 \%)$ participants revealed their friends using injectable drugs. A total of $33(6.73 \%)$ participants expressed their involvement in sexual relation, of which $29(29 / 215,13.49 \%)$ were boys and $4(4 / 275,1.45 \%)$ were girls $(p<0.01)$ (Table 2$)$. The first sexual encounter before the age of 16 years was seen in $7(7 / 117,5.98 \%)$ participants, whereas in $26(26 / 373,6.97 \%)$ participants the first sexual encounter was seen after the age of 17 years (Table 3 ). There was no significant difference in the first sexual encounter with respect to age as well as sex. All sexually engaged participants had heterosexual relation. None of the participants said they were involved with multiple sexual partners simultaneously, but $13(13 / 33,39.39 \%)$ participants expressed their involvement with multiple sexual partners at different periods of life. Multiple sexual partners were seen only in participants belonging to the age group of $\geq 18$ years; $1 / 16(16.67 \%)$ in the age group of 18 years and $12 / 26(46.15 \%)$ in the age group of 19 years $(p=0.50)$. Most boys $(12 / 29$, $41.38 \%)$ were involved with multiple sexual partners than girls $(1 / 4$, $25 \%)$, however, this was not statistically significant $(p=0.50)$. Out of 33 participants involved in sexual relation, only 16 (48.49\%) participants had taken protective measures against sexually transmitted diseases and all were boys. None of the girls provided history of their partner taking any protective measures.

\section{DISCUSSION}

In this study, we performed serological tests to estimate the prevalence of blood-borne viral infections, as it possible to directly evaluate the 
Table 1: Age and sex distribution of participants

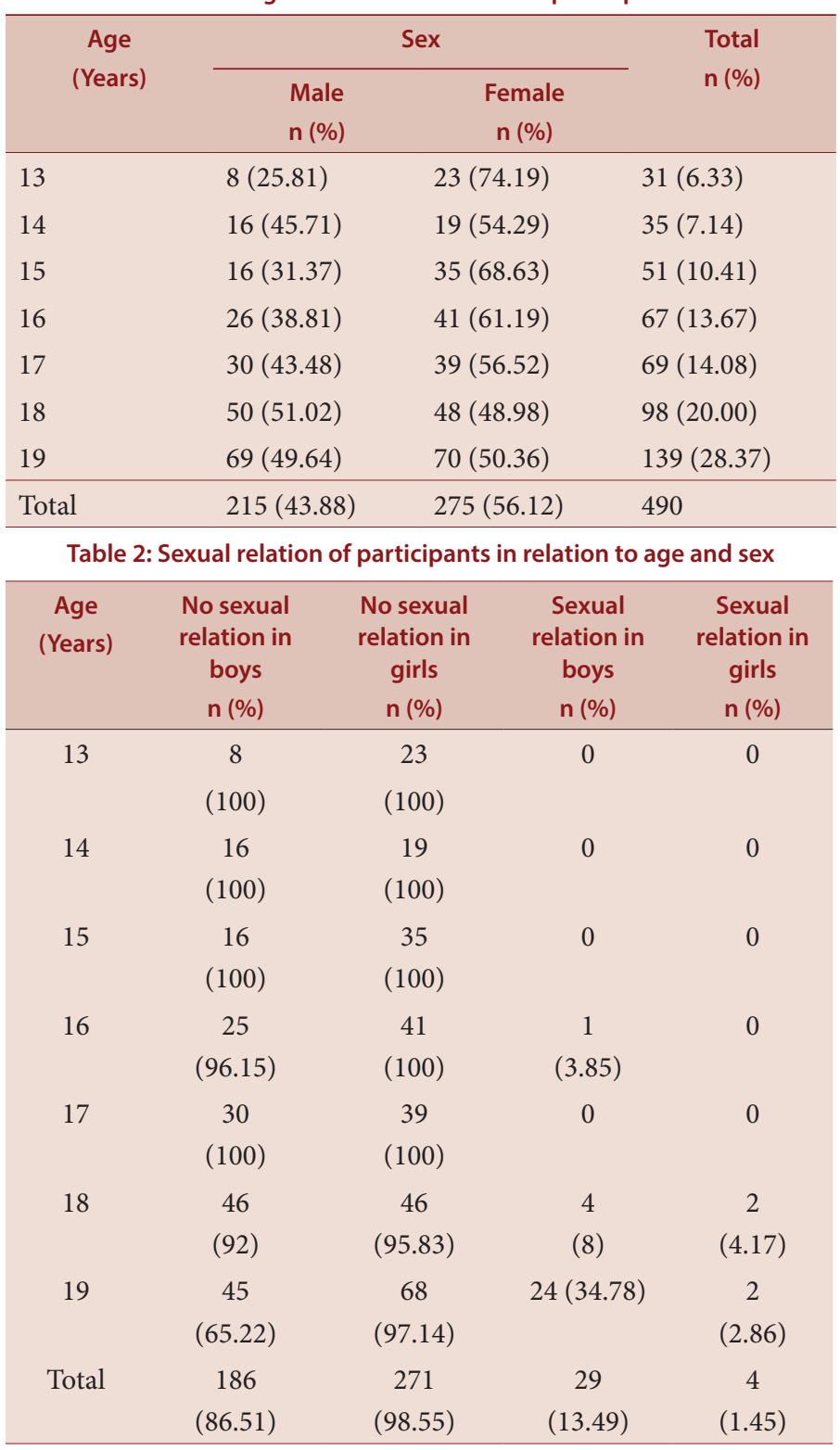

prevalence of HIV, HBV, and HCV infections in a population. We studied adolescent population from eastern part of Sikkim, as it is the most populated area in Sikkim according to the Census 2011 conducted by the Census Organization of India. ${ }^{13}$ The absence of any program designed to study the prevalence of a given condition in our country makes it difficult to reach the entire population. The best way to enroll adolescents for a study is via schools and colleges; therefore, our study involved participants from schools and colleges who are living in both urban as well as rural areas. Therefore, the population in this study is a selected one and does not reflect the general adolescent population of our state. Because the participants in the class were not according to the age appropriate for the class, they were much older than they should have been. Therefore, an equal number of participants in each of the age group could not be collected for the study.

Though the epidemiology of HIV in India has been extensively studied by the NACO, India, the epidemiology of other blood-borne viruses such as HBV and HCV has not been studied systematically. HBV and HCV infections are expected to ride piggy-back on HIV epidemic and are bound to be a significant cause of morbidity in India. Our study revealed
Table3: First exposure of participants to sexual relation

\begin{tabular}{|cccc|}
\hline $\begin{array}{c}\text { Age } \\
\text { (Years) }\end{array}$ & $\begin{array}{c}\text { Number of boys } \\
\mathrm{n}(\%)\end{array}$ & $\begin{array}{c}\text { Number of } \\
\text { girls } \\
\mathrm{n}(\%)\end{array}$ & $\begin{array}{c}\text { Total } \\
\mathrm{n}(\%)\end{array}$ \\
\hline Before 13 & 2 & 1 & 3 \\
& $(6.9)$ & $(25)$ & $(9.09)$ \\
13 & 2 & 0 & 2 \\
& $(6.9)$ & & $(6.06)$ \\
14 & 0 & 0 & 0 \\
15 & 2 & 0 & 2 \\
16 & $(6.9)$ & & $(6.06)$ \\
& 8 & 2 & 10 \\
17 & $(27.59)$ & $(50)$ & $(30.30)$ \\
& 7 & 0 & 7 \\
18 & $(24.14)$ & & $(21.21)$ \\
& 4 & 0 & 4 \\
19 & $(13.79)$ & & $(12.12)$ \\
& 4 & $(25)$ & $(15.15)$ \\
\hline
\end{tabular}

an absence of HIV and HCV prevalence, however, a very low prevalence of HBV $(0.2 \%)$ in adolescents of east Sikkim was observed. Based on HIV Sentinel Surveillance 2008-2009, it is estimated that 23.9 lakh people are infected with HIV in India. As per United Nations International Children's Emergency Fund (UNICEF), India (2013), the prevalence of HIV in India among $15-19$ year olds is $0.04 \%$. As per Sikkim AIDS control society, Government of Sikkim, India, till date a total of 13 people have died due to HIV/AIDS in Sikkim, whereas 213 people are suffering from HIV/AIDS. Though the number of new annual HIV infections has declined during the last decade, some low-prevalence states have shown a slight increase in the number of new infections over the past 2 years, thus underscores the needs for a program to focus more on the states with low but high vulnerability. ${ }^{14}$ Prevalence of HBsAg in India ranges from 1 to $13 \%$ with an average of $4.7 \%{ }^{15,16}$ High prevalence rates of HBsAg has been noted amon $g$ the Indian tribal population. ${ }^{17}$ Another study revealed that the true prevalence of $\mathrm{HBV}$ carriers in India is likely to be between 1 and $2 \%$ except certain high prevalence pockets such as blood donation and antenatal clinics, which accounts for HBs Ag positivity ranging from 0.7 to $7 \% .{ }^{18}$ Various studies show different prevalence rate of $\mathrm{HCV}$ in different parts of the country. A study from Maharashtra reported very low prevalence of $\mathrm{HCV}(0.09 \%),{ }^{19}$ whereas a study from West Bengal and two studies from Andhra Pradesh reported prevalence of $\mathrm{HCV}$ to be $0.07 \%$ and 1.4 and $2.02 \%$,respectively. ${ }^{20-22}$ However, very high prevalence of $\mathrm{HCV}$ (7.89\%) has been reported from Arunachal Pradesh..$^{23}$ Due to the paucity of data on the prevalence of these bloodborne infections in adolescent population from other parts of the country, it could not be said that the prevalence shown by our study is similar or lower than that of other parts of India.

In our population, the common mode of transmission would be via tattoo/body piercing, invasive (medical) procedure, sharing of personal item, heterosexual transmission, and blood transfusion in the descending order. Multiple sexual partners (39.39\%) and non-use of condom (51.51\% in boys and $100 \%$ in the sexual partners of girls) noted in our study could further increase the risk of transmission of these bloodborne diseases in the study population. Though injectable drug use was not noted in the study population, $0.82 \%$ of the participants revealed their friends using injectable drugs, which suggest that injectable drugs 
could also be one of the modes of transmission of these infections in our population.

This study suggests that this is the right time to create awareness about the blood-borne diseases, risk associated with their transmission, existence and importance of $\mathrm{HBV}$ vaccine, and about their preventive measures in Sikkim. Simultaneously, state-wise systematic survey in diverse sectors of the population should be encouraged to explore the prevalence of these blood-borne diseases in low-risk, high-risk, as well as in general population. In the era when we know the diagnostic as well as the preventive measures for these blood-borne viruses, the prevalence of these blood-borne viruses should not come as shocking news as it happened in Manipur in 1991.

\section{CONCLUSION}

To the best of our knowledge, this is the first study evaluating the adolescent population in Sikkim for blood-borne infections. Our study indicates a very low prevalence of HBV and absence of HIV and HCV infections in adolescent population in east Sikkim. Furthermore, a larger epidemiological study needs to be performed to know the true prevalence rates of these infections in adolescent population in east Sikkim. The risk factors for acquisition of these viruses exist in our population. Multiple sexual partners and non-use of condom could further increase the risk of transmission. Furthermore, injectable drug could also be one of the modes of transmission of blood-borne viruses in our population. Therefore, the population of Sikkim must be made aware of blood-borne infections, risk associated with their transmission, existence of HBV vaccine, and preventive measures for these infections.

\section{ROLE OF THE FUNDING SOURCE}

The sponsor of the study had no role in study design, data collection, data analysis, data interpretation, or writing of the manuscript. The corresponding author had full access to all the data in the study and had final responsibility for the decision to submit for publication.

\section{REFERENCES}

1. Joint United Nations Programme on HIV/AIDS (UNAIDS). 2006 report on the global AIDS epidemic. Geneva, Switzerland: UNAIDS; 2006. Available at http:// www.unaids.org/en/hiv data/2006global report/default.asp.

2. Joint United Nations Programme on HIV/AIDS (2010). 'Overview of the global AIDS epidemic'UN report on the global AIDS epidemic 2010.Commission on AIDS in Asia (2008). Redefining AIDS in Asia: crafting an effective response.
New Delhi, Oxford University Press.

3. Mirante E. Drug Injecting in Manipur, India. The Burma Connection. AIDS \& Society 1993; 4(2): 4-6.

4. Sehgal PN. Shocking findings in Manipur. Health Millions 1991; 17 (4): 26-28.

5. Lee WM. Hepatitis B virus infection. N Engl J Med 1997; 337: 1733-45.

6. Lavanchy D. Public health measures in the control of viral hepatitis: A world health organization perspective for the next millennium. J Gastroenterol Hepatol 2002; 17:452-59.

7. Chowdhury A. Epidemiology of hepatitis B virus infection in India. Hep B Annual 2004; $1: 17-24$

8. Proceedings of the International Workshop on Epidemiology, Diagnosis and Management of Hepatitis C Infection. Medicine and the Community 1996, 6-7:1-32.

9. Census of India 2011. Available at http://www.imaginmor.com/census-of-india-2011.html. (Last accessed 15 April 2014).

10. Sravya K, Madhu V, Meenakshi R, and Sreenivas V. Adolescents living with HIV: An Indian Profile. AIDS Research and Treatment 2012;576:7-10.

11. Guidelines for HIV Testing, March 2007. Available at http://www.naco.gov.in/ NACO/About_NACO/Policy_Guidelines.pdf (last accessed 1st March 2014). (Last accessed 1 February 2014).

12. Population.com: Population of Sikkim-2011census. Available at:www.populationindia.com/2012/03/population-of-sikkim-2011-census.html. (last accessed 15 February 2014) http://aidsdatahub.org/dmdocuments/NACO_Annual_Report_2011_12.

13. Thyagarajan SP, Jayaram S, Mohanavalli B. Prevalence of HBV in general population of India. In: Sarin SK, Singhal AK, editors. Hepatitis B in India: Problems andprevention. New Delhi: CBS; 1996. p. 5-16.

14. Sobeslavsky O. Prevalence of markers of hepatitis B virus infection in various countries: a WHO collaborative study. Bull World Health Organ 1980; 58: 621-8.

15. Prasad SR, Rodrigues FM, Dhorje SP, Ramamoorthy CL. Prevalence \& subtypes of hepatitis B surface antigen in the tribal population of Arunachal Pradesh, India. Indian J Med Res 1983;78:300-6.

16. Lodha R, Jain Y, Anand K, Kabra SK, Pandav CS. Hepatitis B in India: A Review of Disease Epidemiology. Indian Pediatr. 2001;38:349-71.

17. Chadha MS, Tungatkar SP and Arankalle VA. Insignificant prevalence of antibodies to hepatitis C in a rural area of western Maharashtra. Indian J Gastroenterol 1999; 18: 22-23.

18. Chowdhury A, Santra A, Chaudhuri $S$ et al. Hepatitis $C$ virus infection in the general population: a community-based study in West Bengal, India. Hepatology 2003;37:802-9.

19. Chandra $\mathrm{M}$, Khaja $\mathrm{MN}$, Farees $\mathrm{N}$ et al. Prevalence, risk factors and genotype distribution of HCV and HBV infection in tribal population: a community based study in south India. Trop Gastroenterol 2003;24:193-5.

20. Khaja MN, Madhavi C, Thippavazzula R et al. High prevalence of hepatitis $C$ virus infection and genotype distribution among general population, blood donors and risk groups. Infect Genet Evol 2006;6:198-204.

21. Phukan SS, Das HK, Mahanta J. HCV activity in an isolated community in north east India. Indian J PatholMicrobiol 2001;44:403-5. 